\title{
Remote delivery of cognitive science laboratories: A solution for small disciplines in large countries
}

\author{
MICHAEL KALISH and STEPHAN LEWANDOWSKY \\ University of Western Australia, Nedlands, Australia \\ and \\ SIMON DENNIS \\ University of Queensland, Brisbane, Queensland, Australia
}

\begin{abstract}
Numerically small disciplines can be jeopardized by geographic difficulties, if student populations at universities are small and distances between them large. This problem could be addressed, if teaching resources could be shared among several universities and students in several locations could be taught simultaneously. We present the results of a trial in simultaneous Internet and videoconferencing delivery of an introductory cognitive science laboratory. The trial relied on off-the-shelf software and hardware. Students found the delivery nearly as effective as a locally run laboratory, despite a noticeable difference in the level of interactivity between the remote and other, local laboratory classes. We discuss possible further improvements in teaching efficiency and efficacy.
\end{abstract}

In an era of increasingly scarce resources, numerically small disciplines often have to rely on collaboration between universities to remain viable. One form or such cooperation is to have agreements whereby only one of several nearby universities offers a program in cognitive science, meteorology, or other small disciplines. Students from other universities could then reciprocally enroll at these universities. This solution can be unavailable if universities are separated by large geographic distances. In the case of Australia, with a population of only 18 million people spread over a country roughly the size of the continental United States, distance has created particular difficulties for small disciplines such as cognitive science. Although Australia represents an extreme case of a sparsely populated country with vast distances between metropolitan centers, geographic difficulties are of universal concern to small disciplines in large countries.

These geographic difficulties may be overcome by the use of the Internet and related technologies to increase the reach of a single instructor, so that students in several locations can participate remotely in a single class or laboratory. If remote classes can be as satisfying for students as local ones, remote delivery can be a genuine aid to struggling programs. Furthermore, it may be undesirable to eliminate small programs, because of staffing issues, for example. This article reports the results of a remote de-

This research was supported by a grant from the Teaching and Learning Center at the University of Western Australia to the first two authors. Thanks to Roy Ebert and an anonymous reviewer for helpful comments on an earlier draft. Correspondence concerning this article should be addressed to M. Kalish, Department of Psychology, University of Western Australia, Nedlands, 6907, Australia (e-mail: kalish@ psy.uwa.edu.au/user/kalish). livery trial, in which two distant universities cooperated to share a single laboratory in cognitive science.

Remote delivery can take many forms. Instructional materials can be delivered remotely, either to individual students or to a group of students who share a common facility. The Internet has proven very useful for delivering instruction to individual students, and a variety of applications have revealed important considerations for curriculum development (see, e.g., Abrahamson, 1998; Dirks, 1997). Similarly, distance instruction of traditional classroom groups has been conducted with some success in academic (see, e.g., Furnace, Hamilton, Helms, \& Duguid, 1996; Kirstein \& Beckwith, 1991) and business training (see, e.g., Pugh, Parchman, \& Simpson, 1992) environments. Unlike the individual student model, distance education with groups typically entails audiovisual communication, rather than relying exclusively on the Internet. Traditional courses that have used the Internet have, instead, used as it a supplement to the teaching of courses to local students. A number of effective models and design considerations have been developed for this supplemental context in which the primary instruction is delivered in a traditional setting (see, e.g., Aberson, Berger, Emerson, \& Romero, 1997; Rosen \& Petty, 1997; Svanum, Chen, \& Bublitz, 1997). As we discuss below, the Internet can also be used to supplement remote delivery to a conventional classroom group, by using the Internet alongside more traditional videoconferencing technology.

\section{Factors in Remote Delivery of Cognitive Science}

The array of remote delivery technologies that are now available is substantial. Three factors that distinguish these technologies include communication mode, channel types, and communication structure. Communication mode differentiates between synchronous and asynchro- 
nous interactions. Synchronous communication (such as videoconference or Internet Relay Chat) has the advantage of immediate feedback, whereas asynchronous communication (such as e-mail or bulletin board) promotes flexibility (Hiltz, 1992). Channel type refers to the attributes of the communication channel (e.g., video, audio, full-screen images, or text). For example, videoconferencing provides both visual and auditory channels, whereas chat provides a text-based read-and-type channel. Finally, communication structure refers to the organization of interactions. The communication may be one way or two way and may have single or multiple users at each end. Common instances are one to one (tutor style), one to many (lecture style), or many to many (discussion style).

The choice of a medium for instruction delivery is partly determined by the nature of the material being communicated. Because of the multidisciplinary nature of cognitive science, instructors inherit a variety of teaching methodologies. In philosophy, the emphasis is on discussion and debate. In computer science, the emphasis is on problem solving, whereas in psychology, it is on observation and experimentation. Similarly, the students that are attracted to cognitive science courses bring a variety of learning styles. Successful flexible delivery of cognitive science will draw on solutions across a variety of delivery options.

\section{Context of Remote Delivery Trial}

A principal requirement of the cognitive science undergraduate program at the University of Western Australia is a one-semester course (i.e., 13 weeks of lectures) in the 2 nd year that requires completion of twelve 2-h laboratories. The laboratory topics include connectionist modeling, expert systems, problem solving, language comprehension, cognitive anthropology, and neuropsychology.

We examined remote delivery of one laboratory on the back-propagation neural network algorithm. During the trial, the instructor was located in Brisbane, Queensland, with the students participating in Perth, Western Australia. The laboratory included a brief lecture to orient the students, followed by a practical tutorial lasting about $1 \mathrm{~h}$. During the trial, the lecture component was used to refresh the students' understanding of the learning algorithm, whereas the tutorial component required the students to work through a set of exercises, using a connectionist simulator. In an earlier laboratory, the students were introduced to our chosen simulator, a Java-based program called BrainWave (co-developed by the third author, S.D.).

We make the assumption that university students typically expect a high level of instructor interaction. In distance learning, this interaction can range from simple email contact through studio-produced, satellite-delivered, multimedia programs (Colbert, Voglimacci, \& Finkelstein, 1995). Hacker and Sova (in press) suggest that simple asynchronous e-mail systems can be an effective teaching aid, but they are unlikely to be a substitute for the interactivity of a live tutor. In trying to construct a tradi- tional classroom experience with a remote instructor, we therefore chose to rely entirely on synchronous delivery.

In terms of communication channel, we determined to adopt as rich a form of communication as possible. Videoconferencing systems are well used and can be effective in delivering lectures (Kirstein \& Beckwith, 1991; Pugh et al., 1992; Whittington, 1987). These systems can be reasonably affordable, although systems of very high quality, which use very large bandwidths to communicate, can be extremely expensive. In this instance, the Internet supplies a constructive support system for a relatively inexpensive, low-bandwidth videoconferencing system.

The BrainWave simulator provides a highly visual interface, much like a drawing program. To exploit this advantage, we created a synchronous Internet link (using Microsoft NetMeeting) that allowed the instructor to control a projected computer display in the remote location. The simultaneous display of computer-based text and graphics with live video represents a highly effective delivery option, previously available only with the aid of a professional production director (Colbert et al., 1995). The synchronous Internet link also resolved a difficulty of conventional videoconferencing systems that rely on a document camera to transmit visual aids, such as overhead transparencies. The poor image quality associated with use of the camera and the difficulty of its operation can give rise to dissatisfaction with videoconferencing (Furnace et al., 1996).

In summary, both videoconferencing and the linked computers are synchronous forms of communication. Both provide visual channels, the videoconferencing provides an additional auditory channel, and the Internet link provides an additional read-type channel. The linked computers and the videoconferencing unit are useful in the one-to-many situation of the lecture and the one-to-one structure of the tutorial. The computer link provides clear lecture slides and the ability to demonstrate the BrainWave software, whereas the videoconferencing unit may provide a familiar interface with the distant instructor.

\section{METHOD}

\section{Subjects}

Twenty-five University of Western Australia (UWA) students who were enrolled in the introductory cognitive science class participated as part of their course requirement. Two identical replications of the laboratory were conducted on consecutive days, with about half of the students attending on each day. The laboratory lasted just under $2 \mathrm{~h}$.

\section{Equipment}

The UWA cognitive science teaching laboratory is equipped with a mixture of SGI workstations and Pentium PCs running Windows 95. The BrainWave simulator is a Java program, so both platforms were available for the lab. Communication was simultaneous over the Internet, via Microsoft's NetMeeting, which allows real-time remote control of a PC, and by videoconference, via a 128-Kbit ISDN connection. The ISDN line was divided into three parallel streams; $46.5 \mathrm{Kbit}$ of bandwidth was devoted to audio signal, and $10 \mathrm{Kbit}$ to control information, leaving the balance for video signal. This allowed a frame rate of approximately $4-5$ frames per sec. 
The laboratory was delivered from a videoconferencing studio in Queensland to the teaching laboratory in Western Australia, where the video image was displayed on a videoconferencing system and the computer image was projected onto a large screen. The students therefore had access to two live images, one that displayed the instructor (S.D.) in Queensland and one that displayed his computer interactions.

\section{Procedure}

Communication occurred in two modes, passive and interactive. Initially, the instructor delivered a brief lecture, using the synchronous Internet connection to supply high-quality visuals (PowerPoint slides to accompany the lecture) alongside the lower quality live video. The passive mode permitted limited interactivity, via questions from the audience in Western Australia. The lecture described the limitations of single-layer perceptrons and the perceptron learning algorithm. The need for multilayer networks and the importance of error-driven, supervised learning were described, and the backpropagation algorithm was presented nonmathematically. Finally, the BrainWave simulator was used to illustrate the operation of the back-propagation algorithm.

Following the lecture, the students participated in a Web-based tutorial on their own, with local tutors handling some of the students' questions but directing others to the remote instructor via the videoconferencing system. The parallel NetMeeting connection gave the remote instructor the ability to access some students' computer screens (those that were working on PCs only), to help with answering of questions. The tutorial included training of an artificial neural network using back-propagation, followed by generation of sets of training patterns. The students used the simulator to answer questions about network weights and activations. Each student was expected to complete the tutorial prior to the end of the laboratory period.

Student reactions to the laboratory were collected twice. A questionnaire was administered 7 weeks after the trial laboratory, at the end of the semester. Free-form comments were generated as (ungraded) homework assignments and were collected the week following the trial. The questionnaire asked the students to compare the experimental laboratory with the average standard laboratory during the semester and contained a set of 14 rating scales. The difference in the interactivity, level of understanding achieved, comfort with the situation, perceived success of the tutorial, and responsiveness of the tutor were measured for the lecture and tutorial portion separately. Overall satisfaction and engagement were also measured, along with students' preferences for the remote versus the live laboratory. Finally, the questionnaire asked students to indicate the proportion of their feelings about the laboratory that was associated with the novelty of the situation.

\section{RESULTS AND EVALUATION}

\section{Technical Aspects}

The 2-h tutorial was completed as planned on both occasions. All the students successfully completed the tutorial exercises. All the analyses are reported collapsed across the two identical replications.

Difficulties were experienced with the videoconferencing system, owing to the periodic breakup of a synthesized ISDN line. ${ }^{1}$ Thus, the system disconnected several times during the laboratory, requiring a redial to reconnect. The Internet connection was vital in maintaining continuity of contact. A chat program was used to provide updates on the technical problems.

\section{Response by Academics}

The laboratory was attended by two or three academics on each of the two occasions. The remote lecture was delivered well, with both the lecturer and the students developing a tolerance to the periodic disconnects. The students had to be encouraged to speak clearly in response to lecturer questions but rapidly learned to do so. The academics on both ends viewed the delivery as effective, owing in large part to the mixture of media (Internet and video). Owing to the limited temporal resolution of the video connection, the high-quality computer visuals delivered by Internet were particularly important. In fact, both the lectures themselves and the students' responses appeared very similar to local, live deliveries.

\section{Response by Students}

Free-form comments were very positive, consistent with the impressions of the academics involved. The students knew that the laboratory was a special event, and so the source of their satisfaction is, of course, open to question. The majority of the students viewed the exercise as a "success," and most found it "exciting."

The results of the rating scale data taken 7 weeks later echoed some of this positive reaction. The questionnaire was divided into three parts, with responses tallied separately for the lecture items, the tutorial items, and the items dealing with the laboratory as a whole. The response to each individual item was made on a 5-point scale, which was coded from -2 to 2 , with -2 meaning a strong preference for the standard laboratory and 2 meaning a strong preference for the trial laboratory. The responses of each student to all the items in each of the sections of the questionnaire were averaged, producing three scores (one for each section: lecture, tutorial, or overall). The results were analyzed, using a one-way repeated measures analysis of variance (ANOVA), with section serving as the independent variable. There was no significant effect of section $\left[F(2,36)=1.16, M S_{\mathrm{e}}=0.146, p=\right.$ .33]. The students did not feel significantly different about the laboratory overall, as compared with its lecture or tutorial components. Importantly, the mean of the overall responses was positive $(M=0.18)$ and significantly different from zero $\left[F(1,18)=3.95, M S_{\mathrm{e}}=0.471, p=.06\right]$. Thus, the subjects were generally positive about the trial laboratory, finding it slightly better than the average standard laboratory. Although this may reflect on the distance delivery itself, it is also possible that most, or even all, of the individual laboratories would be remembered as being better than the average standard lab. Without asking those 12 additional sets of control questions, we simply conclude that the trial laboratory was not perceived as being significantly worse than the average standard lab.

The nonsignificant difference between parts of the questionnaire allows us to compare the individual aspects of the remote delivery, as probed in the individual items contained in the questionnaire. Because the same five questions were used to rate both the lecture and the tuto- 

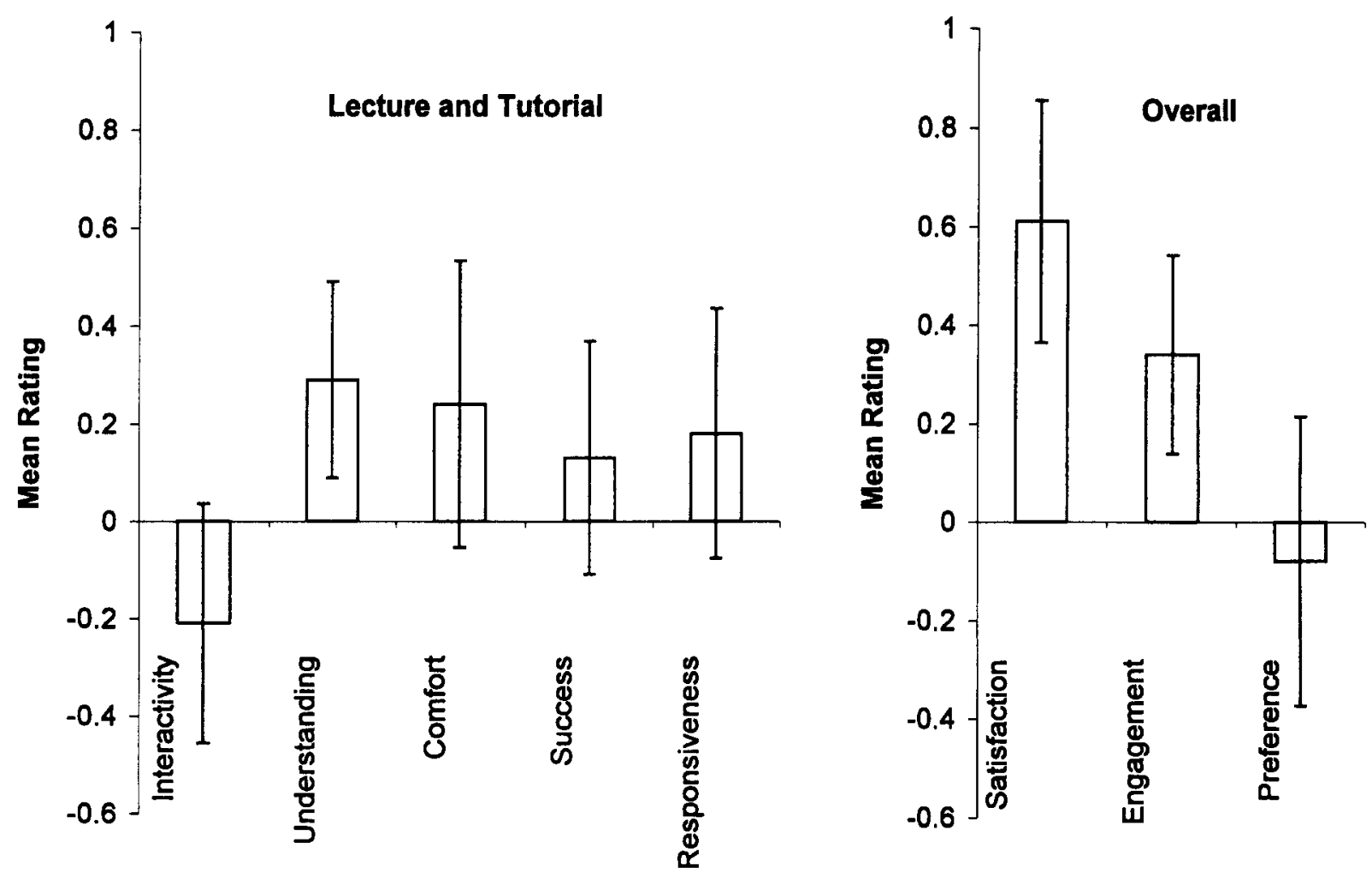

Figure 1. Student perceptions of remote delivery laboratory. Negative ratings indicate that the trial laboratory was perceived as worse than the average standard laboratory on an item. Lecture and tutorial sections were rated separately, and the results were averaged. The students also rated three overall aspects of the laboratory. Error bars show the $95 \%$ confidence interval of the mean rating for each item. Only Understanding (of the lecture and tutorial) and Satisfaction (with the laboratory overall) were significantly different from zero. All $\mathrm{Ns}=19$.

rial portions of the laboratory, the two ratings of each subject were averaged to form a single score for each subject for each of the five questions. The resulting five average ratings per subject were analyzed, using a one-way repeated measures ANOVA, with question as the factor. The effect of question was significant $\left[F(4,72)=5.10, M S_{\mathrm{e}}=\right.$ $0.145, p<.001]$. As is shown in Figure 1, the students judged all aspects of the trial laboratory positively, with the exception of the Interactivity item. Only the positive judgment about Understanding was significant, however, with all the other ratings not being significantly different from zero. Thus, despite the fact that the lecture and the tutorial were marginally less interactive than the standard laboratory, the students did not feel negative about any part of the instruction and felt positive about the understanding they achieved for the topic of the laboratory.

The students' ratings of the overall laboratory (as given in the third section of the questionnaire) were also significantly positive, as is shown in Figure 1. No strong preference for either live or remote laboratories was recorded, despite the fact that the students were significantly more satisfied by the trial laboratory than they were for the average standard laboratory.

Finally, we asked the students to indicate how much of their feeling about the laboratory had to do with its nov- elty. The students reported that more than half of their positive feelings $(M=67.8 \%, S D=15.6 \%)$ were due to novelty, although the question did not supply an alternative source for these feelings. This attraction to novelty, even in the face of technical difficulties, has also been observed in other traditional-group distance education reports (Furnace et al., 1996).

\section{DISCUSSION}

In essence, the remote delivery laboratory functioned at least as effectively as a live-delivery laboratory. This suggests, then, that departments at distant universities can potentially increase the breadth of their offerings by using each other's expertise in the teaching of specialized subjects.

In the trial we presented, we combined videoconferencing with a synchronous Internet connection. This provided an opportunity to observe the operation of these two communication channels working in tandem. It is noteworthy that, although the videoconferencing system was potentially the most expensive component of the trial, it provided the least robust delivery. At the same time, the students' free-form reports made it clear that the videoconferencing was the most interesting part for the sub- 
jects. Likewise, the academic staff felt that the videoconferencing was vital for maintaining a sense of connection with the distant instructor.

We used a relatively affordable videoconferencing delivery method, in which an ISDN line carried video and audio from the lecturer to the students and back. Because of the low bandwidth on the line, the frame rate was relatively low, although audio quality was very high. There were only very brief lags between the sites, making twoway audio communication possible. Accordingly, the students found the interactivity of the presentation to be only marginally, if at all, below that of a live laboratory. A more expensive option would be to use a higher bandwidth, as is typical in teleconferencing-only distance education (Colbert et al., 1995; Hansford \& Baker, 1990; Kirstein \& Beckwith, 1991). Although such a system might increase the perceived interactivity of the lecture phase of our laboratory, there is little reason to suppose that it would alter either the interactivity of the tutorial phase or the overall quality of instruction. The students were satisfied with the trial as we delivered it, despite the perception of lower-than-normal interactivity. An increase in bandwidth is probably not the best way to increase the perceived interactivity of the trial.

One possible way to improve the delivery of the laboratory would be to increase the ease with which students interact with the remote tutor. Although the student ratings did not reveal any difference between the interactivity of the lecture and the tutorial phases, the observations of the academics suggest otherwise. Recall that live tutors were available locally during the tutorial phase and that students had to be encouraged to use the videoconferencing system to communicate with the remote lecturer. Studies have suggested that familiarity with videoconferencing can make the interactions with remote participants much more like familiar face-to-face communication (Colbert et al., 1995; Furnace et al., 1996). If this is the case, increased familiarity with the videoconferencing system through pretraining, for example-might improve student perceptions of the laboratory as a whole.

Other alternatives for delivery certainly exist, but one of our primary concerns was to maintain the laboratorygroup experience as it exists in our standard classes. One reason is pedagogical. The interaction students have with each other during the tutorial is an important part of their learning process (Abrahamson, 1998). The other reason is practical. The purpose of our distance delivery was to increase the reach of individual instructors. Alternatives that allow flexible delivery to individual students would require, at the least, a substantial course redesign; the existing instructional design is suitable for synchronous communication only.
Our goal was to test a technology that allows the simultaneous delivery of live and remote laboratories between cooperating universities. Such a technology allows us to export our teaching expertise with a minimum of reorganization of courses, requiring only that students at remote universities use the same basic set of laboratory exercises. The combination of Internet and teleconferencing appears to offer an effective method, with only limited initial capital investment.

\section{REFERENCES}

Aberson, C. L., Berger, D. E., Emerson, E. P., \& Romero, V. L. (1997). WISE: Web Interface for Statistics Education. Behavior Research Methods, Instruments, \& Computers, 29, 217-221.

ABRAHAMSON, C. E. (1998). Issues in interactive communication in distance education. College Student Journal, 32, 1998.

Colbert, M., Voglimacci, C., \& Finkelstein, A. (1995). Live, audio-visual communication systems for distance learning: Experience, heuristics and ISDN. Behaviour \& Information Technology, 14, 267-288.

Dirks, M. (1997). Problems, philosophy and motivation: Trail blazing instructors for the digital frontier. Journal of Educational Computing Research, 17, 47-65.

Furnace, J., Hamilton, N. M., Helms, P., \& Duguid, K. (1996). Medical teaching at a peripheral site by videoconferencing. Medical Education, 30, 215-220.

HACKer, R., \& Sova, B. (in press). Flexible learning in a partnership context for beginning teachers. Journal of the Association for Learning Technology

HANSFORD, B., \& BAKER, R. (1990). Evaluation of a cross-campus interactive video teaching trial. Distance Education, 11, 287-307.

HILTZ, S. R. (1992). Constructing and evaluating a virtual classroom. In M. Lea (Ed.), Contexts of computer-mediated communication. New York: Harvester Wheatsheaf.

KIRSTEIN, P., \& BECKWITH, R. (1991). Experiences with the University of London interactive video education network. (Research note RN/91/10). University College London, Department of Computer Science.

Pugh, H., Parchman, S., \& Simpson, H. (1992). Video telecommunications for distance education: A field survey of systems in the US public education, industry and the military. Distance Education, 13, 43-64.

Rosen, E. F., \& PETTY, L. C. (1997). Using Internet resources in a research methods course. Behavior Research Methods, Instruments, \& Computers, 29, 222-227.

Svanum, S., Chen, S. H. A., \& Bublitz, S. (1997). Internet-based instruction of the principles of base rate and prediction: A demonstration project. Behavior Research Methods, Instruments, \& Computers, 29, 228-231.

WhitTiNGTON, N. (1987). Is instructional television educationally effective? A research review. American Journal of Distance Learning, 1, 47-57.

\section{NOTE}

1. The commercial carrier we used (Telstra) had just implemented a new software ISDN switching scheme the week of our trial. Most ISDN lines would be more reliable than the service we received.

(Manuscript received October 27, 1998; revision accepted for publication February 5, 1999.) 